\title{
EDITORIAL
}

\section{Lung hyperinflation in airway obstruction}

\author{
V. Brusasco*, J-W. Fitting ${ }^{+}$
}

Since the early studies with inert gas dilution techniques, it has been known that residual volume and functional residual capacity are increased in patients with pulmonary emphysema [1] or acute asthma [2]. These findings were successively confirmed in studies using gas dilution techniques as well as plethysmography or lung imaging. Despite considerable interest on changes in lung volumes during bronchoconstriction, at present there is no consensus on whether the term "hyperinflation" should be used to indicate an increase of functional residual capacity or also of residual volume and total lung capacity.

Hyperinflation in patients with chronic airway obstruction may result either from loss of lung elastic recoil (static hyperinflation) or decreased expiratory flow (dynamic hyperinflation). Understanding the major mechanism for hyperinflation in individual patients may be important for the clinician in order to predict the potential benefits of bronchodilator therapy or optimize the ventilatory support therapy. The mechanisms leading from airway narrowing to hyperinflation will be examined in detail in one of the articles of a new series starting in this issue of the Journal.

Although airway narrowing is the primary abnormality in patients with bronchial asthma or chronic obstructive pulmonary disease, hyperinflation may represent the major cause of symptoms as breathing at increased lung volumes burdens the respiratory system. However, increasing lung volume is in some instances necessary for maintaining adequate minute ventilation and even beneficial in that it increases airway calibre.

Pulmonary hyperinflation markedly alters the shape of the thorax and thereby induces a complex modification of the geometry and interaction of the respiratory muscles. The domain of respiratory muscle physiology is peculiar for its long history. For instance, much of respiratory muscle anatomy and function has been known

*Dept di Science Motorie, University of Genova, I-16132 Genova, Italy. +Division de Pneumologie, Centre Hospitalier Universitaire Vaudois, CH-1011 Lausanne, Switzerland. since Galen (129-200 AD), and modern diagrams of diaphragmatic and rib cage-abdominal motion appear to duplicate those drawn by Leonardo da Vinci (1452-1519) $[3,4]$. In spite of this long standing background, respiratory muscle function and dysfunction remains incompletely understood and has been the focus of intense research during the last 20 years. This has led to a better knowledge of the numerous and complex consequences of hyperinflation on the diaphragm and the intercostal and abdominal muscles. The adaptation of the diaphragm to chronic hyperinflation has received great attention and is not definitively solved in patients. These questions have recently gained a renewed relevance through the development of procedures which attenuate or even suppress hyperinflation, such as lung transplantation or lung volume reduction surgery. The question of how far and fast the thorax and the respiratory muscles will adapt to smaller lungs are yet to be solved. Two future reviews in this series will be devoted to the consequences of hyperinflation on the respiratory muscles and represent the state of the art in this field.

\section{References}

1. Hurtado A, Kaltraider NL, Fray WW, Brooks WDW, McCann WS. Studies of total lung capacity and its subdivisions. VI: Observations on cases of obstructive pulmonary emphysema. J Clin Invest 1934; 13: 1027-1051.

2. Hurtado A, Kaltraider NL. Studies of total lung capacity and its subdivisions. VII. Observations during the acute respiratory distress of bronchial asthma and following the administration of epinefrine. J Clin Invest 1934; 13: 1053-1062.

3. Derenne JO, Whitelaw WA. An abbreviated history of the respiratory muscles from Antiquity to the Classical Age. In: Roussos C, ed. The Thorax. Marcel Dekker, New York, 1995; part A: pp. 399-411.

4. Mitzner WA. Leonardo and the physiology of respiration. In: Proctor DF, ed. A history of breathing physiology. Marcel Dekker, New York, 1995; pp. 37-59. 